\title{
SIMPLIFIED METHOD FOR DETERMINING THE RESISTANCE TO GROUND OF POWER TRANSMISSION TOWERS
}

\author{
A.A. Zaky ${ }^{1}$ and M. T. El-Sonni ${ }^{2}$ \\ 1 Electrical Engineering Department, University of Alexandria, Egypt \\ 2 Computer Engineering Department, Arab Academy for Science and Technology, Egypt
}

\begin{abstract}
The resistance to ground of towers in power transmission lines with ground wires is calculated using difference equations and using the concept of characteristic impedance of a two-port network. The simplicity and elegance of the latter approach is clearly demonstrated, and with practically feasible network terminations is valid for any length of line.
\end{abstract}

Keywords: Tower-footing Resistance, Ladder Networks, Characteristic Impedance.

\section{INTRODUCTION}

In overhead power transmission lines every tower must be grounded such that its standalone resistance to ground does not exceed 10 ohms. As almost invariably such towers carry one or more ground overhead conductors to shield the line against direct lightning strokes, it is important to know how the presence of these conductors affects the total resistance to ground. This resistance will determine the potential to which the tower structure will rise when a ground fault occurs with possible flashovers at the insulators.

It should be mentioned here that there are very few references $[1,2,3]$ to overhead transmission line design in recent literature. This is because in developing countries these highways for energy transmission have been erected a long time ago and are part of their well-established grid infrastructure system. However, today there are thousands of kilometers of new overhead lines being erected throughout developing and third world countries and it is still important that power engineers be well informed on how characteristics such as the tower footing resistance and overhead ground wires can affect line performance under fault conditions. Figure 1 shows the equivalent 'ground' network in which $R$ represents the stand alone resistance of each tower and $r$ the resistance of the ground wire between consecutive towers. It is clear that the resulting network is a ladder-type network with identical loops. It is required to determine the input resistance as seen by a lightning stroke at the beginning of the line and at some point in the middle of the line.

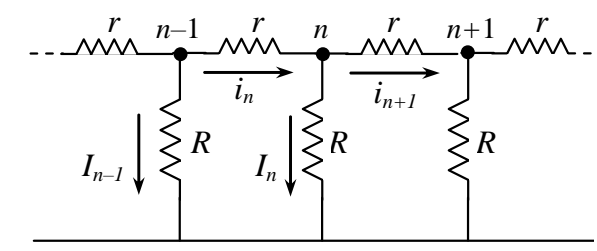

Fig. 1. Equivalent "Ground" Network

In theory any network problem can be solved using the standard methods of circuit theory (mesh and node equations, Thevenin's theorem, etc) in conjunction with (where 
necessary) differential or difference equations. The complexity of the mathematics involved is commensurate with the complexity of the network. However, as pointed out by Guillemin [4] it is always advantageous to inspect a network to determine whether its geometry or distribution of parameter values enables one to use a simplified procedure which leads to the correct required solution. In the following analysis we shall demonstrate this by deriving the required input resistances at the points of fault using a standard procedure and using a simplified procedure. In the analysis it is assumed that all towers have the same resistance to ground. Since tower and ground wire resistances are much larger than their respective inductances, the latter can be neglected. However should it be necessary to take inductance into account, tower impedance $Z$ and ground wire impedance $z$ could be simply substituted for $R$ and $r$ in all the equations below.

\section{DIFFERENCE EQUATION}

The standard procedure for solving laddertype networks is by using difference equations $[5,6]$. In Fig. 1 each node represents the location of a tower; $n=n$ is the $n$-th tower and $n=0$ is the tower at which the fault occurs. Applying Kirchhoff s current law at the $n$-th node we have that,

$$
I_{n}=i_{n}-i_{n+1}
$$

And applying Kirchhoffs voltage law around the $n$-th mesh gives,

$$
\begin{aligned}
& I_{n} R-I_{n-1} R+i_{n} r=0 \\
& i_{n}=\frac{R}{r}\left(I_{n-1}-I_{n}\right) \\
& i_{n+1}=\frac{R}{r}\left(I_{n}-I_{n+1}\right)
\end{aligned}
$$

substituting (2) and (3) in (1) we obtain,

$$
I_{n+1}-\left(2+\frac{r}{R}\right) I_{n}+I_{n-1}=0
$$

This is a second order difference equation whose general solution is given by,

$$
I_{n}=A e^{\alpha n}+B e^{-\alpha n}
$$

where $A$ and $B$ are constants determined by the terminal conditions.

Substituting (5) in (4) and simplifying gives,

$\frac{r}{R}=e^{-\alpha}+e^{\alpha}-2$

$\cosh \alpha=1+\frac{r}{2 R}$

Equation(6) may also be written as, $\frac{r}{R}=\left(e^{-\alpha / 2}+e^{\alpha / 2}\right)^{2}=\left(2 \sinh \frac{1}{2} \alpha\right)^{2}$

$\sinh \frac{1}{2} \alpha=\frac{1}{2} \sqrt{\frac{r}{R}}$

For the current in the ground wire we have from (2),

$$
\begin{gathered}
i_{n}=\frac{R}{r}\left[A e^{\alpha(n-1)}+B e^{-\alpha(n-1)}-A e^{\alpha n}-B e^{-\alpha n}\right] \\
=\frac{R}{r}\left[A\left(e^{-\alpha}-1\right) e^{\alpha n}+B\left(e^{\alpha}-1\right) e^{-\alpha n}\right] \\
=a e^{\alpha n}+b e^{-\alpha n}
\end{gathered}
$$

where,

$$
a=\frac{A\left(e^{-\alpha}-1\right)}{r / R} \quad, \quad b=\frac{B\left(e^{\alpha}-1\right)}{r / R}
$$

Substituting for $r / R$ from (6) we obtain,

$$
\begin{aligned}
& a=\frac{A}{1-e^{\alpha}}, \quad b=\frac{B}{1-e^{-\alpha}} \\
& i_{n}=\frac{A}{1-e^{\alpha}} e^{\alpha n}+\frac{B}{1-e^{-\alpha}} e^{-\alpha n}
\end{aligned}
$$

We shall now assume that the line is sufficiently long so that the current at its end is negligibly small. As will be shown later this assumption is justifiable for the vast majority 
of transmission lines. For this assumption $A=0$ so that,

$$
\begin{aligned}
& I_{n}=B e^{-\alpha n} \\
& i_{n}=\frac{B}{1-e^{-\alpha}} e^{-\alpha n}
\end{aligned}
$$

We will next consider two cases,

(a) The fault occurs at the first tower $(n=0)$ :

The fault current $I_{f}$ is given by

$$
I_{f}=I_{o}+i_{1}
$$

From (11),

$$
\begin{aligned}
I_{o} & =B \\
i_{1} & =\frac{B e^{-\alpha}}{1-e^{-\alpha}}
\end{aligned}
$$

so that,

$$
\begin{aligned}
& I_{f}=B\left(1+\frac{e^{-\alpha}}{1-e^{-\alpha}}\right)=\frac{I_{o}}{1-e^{-\alpha}} \\
& I_{o}=I_{f}\left(1-e^{-\alpha}\right)
\end{aligned}
$$

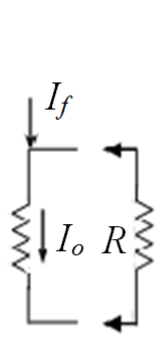

(a)

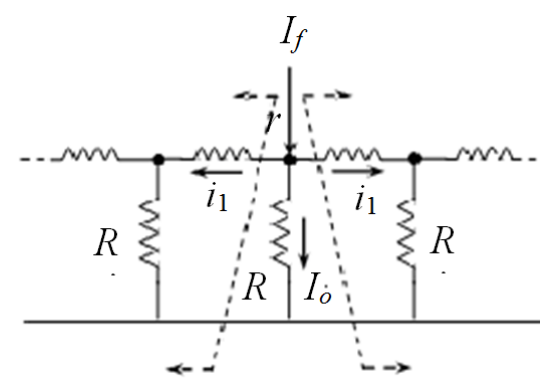

(b)
Fig. 2. The input resistance

(a) At point of fault

(b) At the middle of the line

The input resistance at point of fault is (Fig.2a),

$R_{i}=\frac{R R^{\prime}}{R+R^{\prime}}$

and the current through $R$ is ,

$I_{o}=\frac{I_{f} R^{\prime}}{R+R^{\prime}}=\frac{I_{f} R_{i}}{R}$

Substituting in (13) the input resistance viewed from the line end is,

$R_{i}=R\left(1-e^{-\alpha}\right)$
In terms of the network parameters $R$ and $r$ we have that,

$$
\begin{aligned}
1-e^{-\alpha} & =1-\cosh \alpha+\sinh \alpha \\
& =1-\cosh \alpha+\left(\cosh ^{2} \alpha-1\right)^{1 / 2}
\end{aligned}
$$

substituting from (7) we obtain,

$$
\begin{aligned}
& 1-e^{-\alpha}=-\frac{r}{2 R}+\left[\frac{r}{R}+\frac{r^{r}}{4 R^{2}}\right]^{1 / 2} \\
& R_{i}=\left[r R+\frac{1}{4} r^{2}\right]^{1 / 2}-\frac{1}{2} r
\end{aligned}
$$

(b) The fault occurs at the middle of the line (Fig.2b):

$$
\begin{aligned}
I_{f} & =I_{o}+2 i_{1} \\
& =I_{o}+\frac{2 I_{o}}{1-e^{-\alpha}} e^{-\alpha}=I_{o} \frac{1+e^{-\alpha}}{1-e^{-\alpha}} \\
& =I_{o} \operatorname{coth} \frac{1}{2} \alpha \\
I_{o} & =I_{f} \tanh \frac{1}{2} \alpha
\end{aligned}
$$

The input resistance at point of fault is,

$R_{i}=\frac{R \frac{1}{2} R_{e}^{\prime}}{R+\frac{1}{2} R_{e}^{\prime}}$

and the current through $R$ is ,

$$
I_{o}=\frac{I_{f} \frac{1}{2} R_{e}^{\prime}}{R+\frac{1}{2} R_{e}^{\prime}}=\frac{I_{f} R_{i}}{R}
$$

so that,

$$
R_{i}=R \tanh \frac{1}{2} \alpha
$$

In terms of the network parameters $R$ and $r$ we have that,

$$
\begin{gathered}
\tanh \frac{1}{2} \alpha=\frac{\sinh \frac{1}{2} \alpha}{\cosh \frac{1}{2} \alpha}=\frac{\sinh \frac{1}{2} \alpha}{\left(1+\sinh ^{2} \frac{1}{2} \alpha\right)^{1 / 2}} \\
=\frac{\frac{1}{2} \sqrt{r / R}}{[1+(r / 4 R)]^{1 / 2}} \\
R_{i}=R \tanh \frac{1}{2} \alpha=\frac{r R}{2\left[r R+\frac{1}{4} r^{2}\right]^{1 / 2}}
\end{gathered}
$$

In order to justify the assumption that $A=0$ we will consider some practical orders 
of magnitude. The current in a ground fault is of the order $10^{4} \mathrm{~A}$; if we assume an average span of $250 \mathrm{~m}$ then a $25 \mathrm{~km}$ transmission line (short line) will have 100 towers i.e $n=100$ for a fault at the beginning of the line and $n=$ 50 for a fault at the middle of the line. For $R$ $=10 \Omega$ and $r=1.5 \Omega$, from (7) we have that,

$$
\begin{aligned}
\cosh \alpha & =1+\frac{r}{2 R}=1.075 \\
\alpha & =0.385
\end{aligned}
$$

The current through the towers will be of the order $10^{2} \mathrm{~A}$ and from (5) we have that for $n=0$,

$100=A+B$

And for $n=50$ or $n=100$ the exponential terms in (5) will be respectively of the order $10^{ \pm 8}$ and $10^{ \pm 16}$ so that,

$$
\begin{aligned}
& 100=A 10^{8}+\left(10^{2}-A\right) 10^{-8} \\
& 100=A 10^{16}+\left(10^{2}-A\right) 10^{-16}
\end{aligned}
$$

The orders of magnitude of $A$ are respectively $10^{-6}$ and $10^{-14}$ and hence justifiably negligible. For the above values of $R$ and $r$ we find from equations (15) and (17) that $R_{i}$ is $3.2 \Omega$ at the beginning of the line and $1.9 \Omega$ at the middle of the line.

\section{CHARACTERISTIC IMPEDANCE}

In the simplified procedure we will derive the input resistances using the characteristic impedance of two-port networks. The characteristic impedance $Z_{C}$ of a two-port network is that impedance which when connected to the output terminals will make the input impedance equal to $Z_{C}$.

For a fault at the beginning of the line the repetitive unit is the cantilever circuit formed by $R$ and $r$ (Fig.3) and its characteristic impedance is:

$$
R_{C}=\frac{\left(R_{C}+R\right) R}{R+R_{C}+r}
$$

$$
R_{C}{ }^{2}+r R_{C}-r R=0
$$

In this case $R_{C}$ is also equal to the input resistance,

$$
R_{i}=R_{C}=\left[r R+\frac{1}{4} r^{2}\right]^{1 / 2}-\frac{1}{2} r
$$

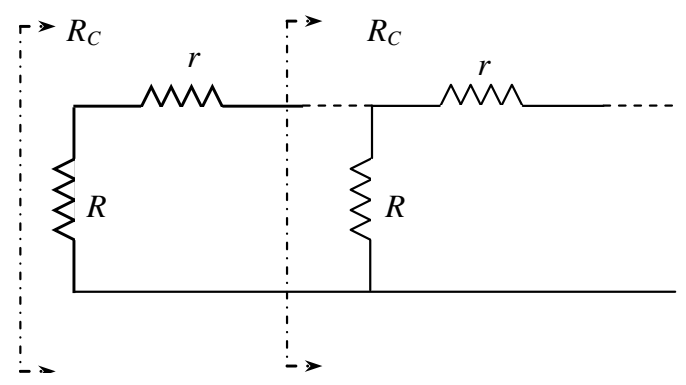

Fig. 3. Cantilever circuit for a fault at the beginning of the line

which is the same as that given in (15).

For a fault at the middle of the line the repetitive unit is the T-network shown in bold in Fig.4. It can readily be shown that its characteristic resistance is,

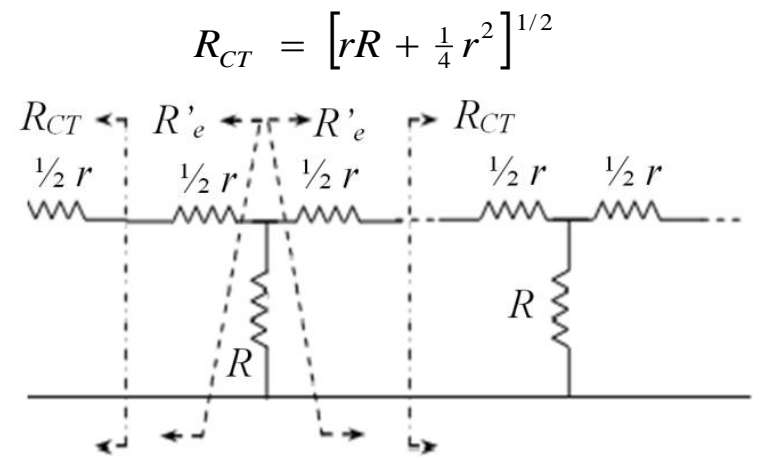

Fig.4. T-Network for a fault at the middle of the line

The input resistance on either side of the faulted tower is,

$$
R_{e}^{\prime}=\left[r R+\frac{1}{4} r^{2}\right]^{1 / 2}+\frac{1}{2} r
$$

If we set

$c=\left[r R+\frac{1}{4} r^{2}\right]^{1 / 2}$ and $d=\frac{1}{2} r$, the input resistance seen by the fault is, $R_{i}=\frac{R \frac{1}{2} R_{e}^{\prime}}{R+\frac{1}{2} R_{e}^{\prime}}=\frac{R(c+d)}{2 R+(c+d)} \frac{(c-d)}{(c-d)}$ 


$$
\begin{gathered}
=\frac{r R^{2}}{2 R(c-d)+r R}=\frac{r R}{2 c} \\
R_{i}=\frac{r R}{2\left[r R+\frac{1}{4} r^{2}\right]^{1 / 2}}
\end{gathered}
$$

which is the same as (17).

If the resistance to ground of the first and last towers (Fig.5), is made equal to

$$
R_{\text {end }}=R_{C T}-\frac{1}{2} r=\left[r R+\frac{1}{4} r^{2}\right]^{1 / 2}-\frac{1}{2} r
$$

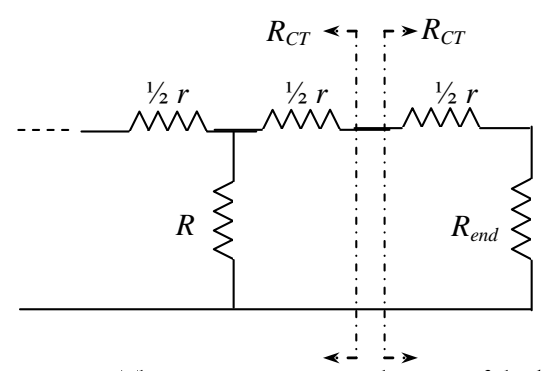

Fig. 5. The input resistance at the start of the line

Then it is evident that the input resistance at any of the towers is,

$$
R_{i}=\frac{r R}{2\left[r R+\frac{1}{4} r^{2}\right]^{1 / 2}}
$$

It may not be so evident that this is also the input resistance at the start of the line. From left to right in Fig. 5 we have that:

$$
R_{i}=\frac{\left(R_{C T}+\frac{1}{2} r\right) R_{\text {end }}}{R_{C T}+\frac{1}{2} r+R_{\text {end }}}
$$

substituting for $R_{\text {end }}$ from (18),

$$
R_{i}=\frac{(c+d)(c-d)}{2 c}=\frac{r R}{2\left[r R+\frac{1}{4} r^{2}\right]^{1 / 2}}
$$

A little consideration will show that although in this case equation (17) as derived from the difference equation remains valid, equation (15) is no longer applicable.

\section{CONCLUSIONS}

A simplified derivation of the total resistance to ground of transmission tower with overhead ground wires has been presented using the characteristic impedance of two-port networks. It is shown that by modifying the footing resistance of the end towers of a line, the input resistance will be equal to that at the middle of the line i.e. effectively reduced by $60 \%$ and hence reduce the risk of flashover for line-to ground faults close to the ends of a line.

\section{REFERENCES}

[1] Endrenyi, J., Analysis of Tower Potentials during ground Faults, IEEE- PAS, 86, 1274-1283, 1967.

[2] Vinton,M., Evaluating Transmission Tower PotentialsDuring Ground Faults, J. Zhejiang University SCIENCA A, Vol.19, 182-189, 2008.

[3] Vinton,M and Buta,A., Ground Fault Current Distribution on Overhaed Transmission Lines, FACT UNIVERSITAT. (NIS) SER: ELEC.ENERG., Vol 19, 71-84, 2006.

[4] Guillemin, E.A., Introductory Circuit Theory, J.Wiley, N.Y., 1953.

[5] Wylie, C.R., Advanced Engineering Mathematics, McGraw-Hill, N.Y.,1966.

[6] Rudenberg, R., Transient Performance of Electric Power Systems, The MIT Press, 1967. 


\title{
طريقة سهلة لتمديد مقاومة أبراج الضغط العالي
}

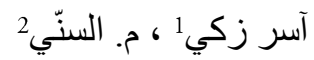 \\ 1 إمعة الإسكندرية، جمهورية مصر العربية \\ 2
}

الملخص:

تقدم هذه الورقة مقارنة بين حساب المقاومة للأرض لأبراج خطوط القوى الكهربية المزودة بأسلاك أرضية علوية باستخدام المعادلات الفرقية وحساب هذه المقاومة باستخدام المعاوقة الخصائصية للاو ائر الثنائية القطب. وتبين هذه الدر اسة أن طريقة المعاوقة الخصائصية تقوم بتبسيط الحسابات بدرجة كبيرة كما تظهر إمكان خفض مقاومة الدخل عند أول الخطوذللك بتحديد قيمة الدقاومة الانفر ادية للأرض لأول وآخر برج من أبر اج الخط. 